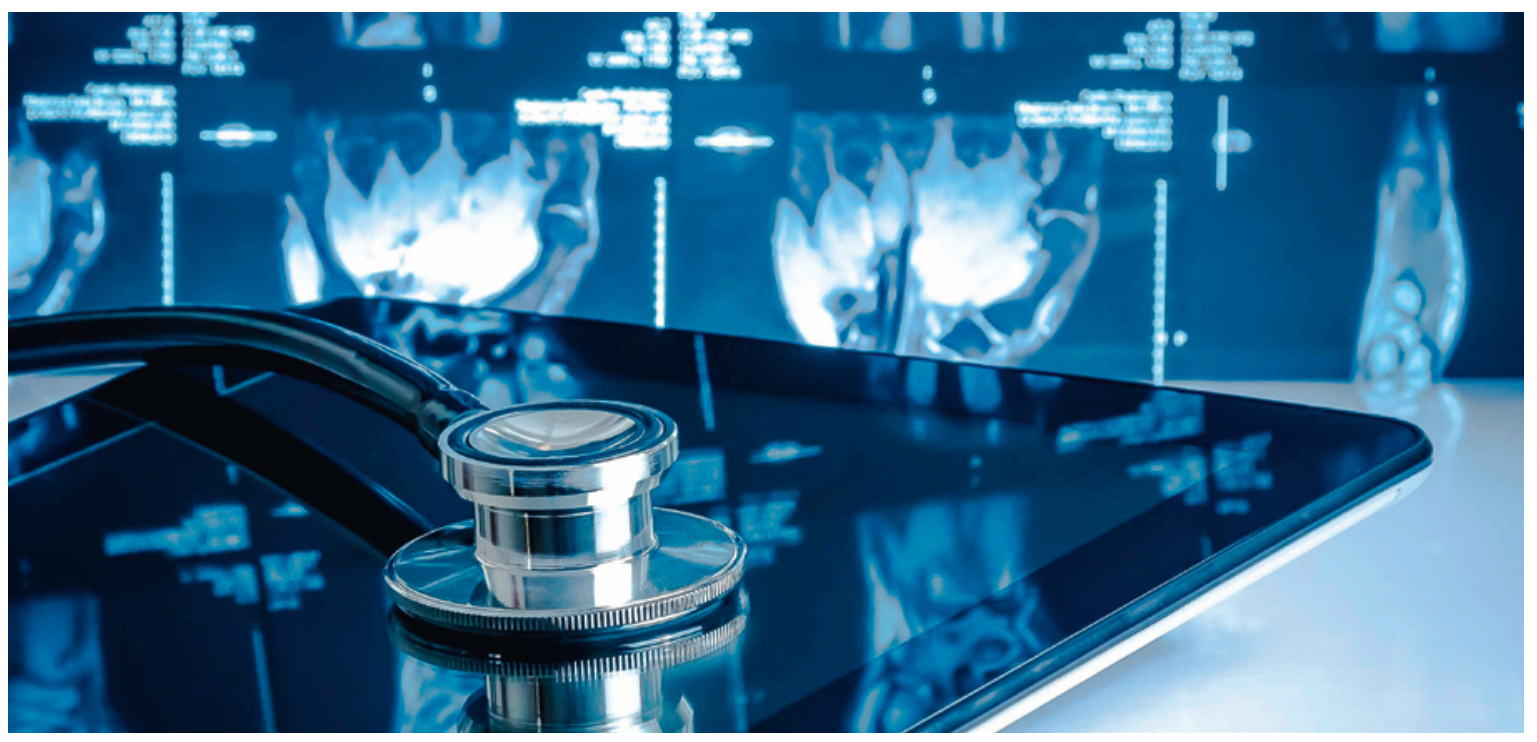

\title{
Zu oft die richtige Diagnose verfehlt
}

\section{Werner Golder}

Prof. Dr. med., Radiologe

\section{Neue Studie bestätigt Zweifel an Teleradiologie}

Das Misstrauen gegenüber der Teleradiologie ist nicht neu. So faszinierend es ist, selbst umfangreiche Datensätze bildgebender Diagnostik jeglicher Modalität mit-

\section{TÉLÉRADIOLOGIE}

La téléradiologie est attrayante, mais potentiellement imprévisible. Une étude publiée dans l'édition de janvier de Clinical Radiology montre que les diagnosticiens à distance se trompent trois fois plus souvent que les médecins sur place lors de l'évaluation de tomodensitométries de l'abdomen. II n'en résulte pas toujours un dommage pour le patient, mais la perte de confiance vis-à-vis du collègue absent est systématique. En contrepartie, de plus en plus de médecins des urgences, qui possèdent de toute manière de très bonnes connaissances de l'imagerie médicale de leur discipline, procèdent eux-mêmes à l'évaluation. La technique médicale leur facilite énormément la tâche au travers des nombreux programmes de traitement des clichés. La radiologie ne peut contrer cette évolution que si ses représentants ont la volonté et la possibilité de se rendre personnellement sur place dans le laps de temps nécessaire pour les soins d'urgence et de participer à la discussion interdisciplinaire. tels elektronischer Übertragungstechnik in kürzester Zeit an jeden beliebigen Ort zu senden, so bestechend es ist, auf diesem Weg konsiliarisch die Meinung von Spezialisten und Subspezialisten zu schwierigen Fragen einzuholen, ebenso problematisch kann der Umgang mit dieser Technik in der klinischen Praxis sein. Als die Teleradiologie vor etwa zwei Jahrzehnten aufkam, setzte man in sie zunächst vor allem die Hoffnung, die in manchen Ländern aufgrund des Mangels an Radiologen zum Teil grotesk langen Wartezeiten bis zur Fertigstellung der kritischen Befundberichte im Routinebetrieb zu verkürzen und andere Versorgungslücken zu decken. Diese Erwartungen waren realistisch und haben sich vielerorts auch erfüllt. Heute aber sind teleradiologische Netzwerke mehrheitlich gleichbedeutend mit der Organisation der fachbezogenen Dienstleistungen bei Nacht sowie an Sonn- und Feiertagen. Die professionelle, in manchen Ländern von international agierenden Teleradiologie-Agenturen mit nicht unbeträchtlichem Erfolg kommerzialisierte Vermittlung sogenannter "Off Site Reporter» für die Interpretation radiologischer Bilddatensätze ist vor allem in den USA, in Grossbritannien und in einigen Stadtstaaten Südostasiens, z.B. Singapur, zu einer prototypi- 
schen Variante des virtuellen Medizintourismus im Katastrophenmodus geworden. Die charakteristischen Schwächen dieses Typs der Notfallradiologie wurden bald erkannt, benannt und zum Teil auch mit statistischen Daten belegt. In der Januar-Nummer von Clinical Radiology - dem offiziellen Organ des "Royal College of Radiologists» - sind nun allerdings die Ergebnisse einer britischen Untersuchung veröffentlicht worden, die die Zweifel nicht nur nachdrücklich bestätigen, sondern manche bisher gehegten Bedenken sogar noch übertreffen [1]. Die Autoren - fünf Radiologen und ein Epidemiologe - haben die Daten von 4931 erwachsenen Patienten ausgewertet, die sich im Jahre 2013 zur Klärung der Ursache nicht traumatischer Bauchschmerzen notfallmässig einer Computertomographie unterzogen haben. Etwas mehr als die Hälfte (52\%) der Untersuchten waren von Ärzten chirurgischer Fachabteilungen überwiesen worden. Als Grenzwert für die Akzeptanz der Fehlerquote im vorläufigen fachärztlichen Befundbericht wurden fünf Prozent definiert. Diese Quote ist von den vor Ort tätigen Radiologen mit 3,1\% ("provisional report») bzw. 2,9\% ("consultant addendum») nicht nur erreicht, sondern sogar weit unterboten worden. Die Tele-Diagnostiker verfehlten das vergleichsweise bescheidene Ziel hingegen deutlich, und zwar bei den Allgemeinpatienten in 8,7\% und bei den chirurgischen Patienten in 12,7\% der Fälle; sie haben sich also etwa dreimal so oft geirrt wie die vor Ort tätigen Ärzte. Die Autoren der Studie versuchten ausserdem, die negativen Folgen (z.B. Fortsetzung der bildgebenden Diagnostik, Endoskopie, Verzögerung der Diagnose/Therapie) der radiologischen Fehldiagnosen zu quantifizieren. Dabei kamen sie auf einen Anteil von 1,0\% in der nicht chirurgischen und 1,5\% in der chirurgischen Klientel.

\section{Welche Folgen zog eine Falschbeurteilung nach sich?}

Man kann zu bedenken geben, dass nicht jede irrige radiologische Aussage zwangsläufig Schaden für den Patienten bedeuten muss, weil das Ergebnis der bildgebenden Diagnostik nur einer von mehreren Faktoren bei der Entscheidung über die Wahl des weiteren diagnostischen und des therapeutischen Vorgehens ist und die Bedeutung, die man ihm beimisst, auch von der Reaktion des behandelnden Arztes auf die Beurteilung des Radiologen bestimmt wird. Die von den Autoren mitgeteilten Prozentzahlen bestätigen die Richtigkeit dieses Einwands: Tatsächlich hat nur etwa jeder zehnte Studienteilnehmer, dessen Bildmaterial falsch beurteilt worden ist, dadurch auch einen Schaden erlitten. Doch es sind noch andere Folgen zu bedenken.
Bereits eine einzige gravierende Fehldiagnose genügt, um das Vertrauen der behandelnden Ärzte in die Kompetenz des Radiologen tief und dauerhaft zu erschüttern. Und die begleitenden Haftungsfragen, die vor allem dann knifflig werden können, wenn der Telediagnostiker im Ausland arbeitet, sind dabei noch gar nicht berücksichtigt.

Es fehlen häufig ausreichende Informationen über vorangegangene bilddiagnostische Massnahmen und die Ergebnisse anderer apparativer Untersuchungen.

Man kann dem Radiologen, der den Befundbericht nicht am Ort der Untersuchung erstellt, zugutehalten, dass er mit der Übernahme jedes einzelnen Auftrags in eine durchaus heikle Situation gerät. Da wird ihm unangekündigt vielfach umfangreiches Bildmaterial vorgelegt, von dem er nicht genau weiss, unter welchen klinischen Umständen es gewonnen wurde. Da wird ihm mit der Überweisung gewöhnlich eine knappe diagnostische Frage gestellt, die die aktuelle klinische Situation des Patienten oft nur unvollständig beschreibt. Da fehlen häufig ausreichende Informationen über vorangegangene bilddiagnostische Massnahmen und die Ergebnisse anderer apparativer Untersuchungen (z.B. Labordiagnostik). Und da befindet sich der Telediagnostiker grundsätzlich unter einem enormen Zeitdruck, der noch dramatisch zunehmen kann, wenn der nächste Auftrag bereits in der Leitung wartet. Fachleute wie Laien können nachvollziehen, dass der Radiologe unter diesem Druck den einen Befund übersieht und den anderen überschätzt, eine (zu) lange bzw. (zu) wenig an die aktuelle Situation angepasste Liste von Differenzialdiagnosen erstellt, nähere diagnostische Festlegungen vom Vergleich mit Voraufnahmen und dem Abgleich mit den Resultaten anderer diagnostischer Verfahren abhängig macht und dabei u.U. mehr Folgediagnostik empfiehlt, als erforderlich ist. Solche Abwehrreflexe sind für die Teleradiologie nicht spezifisch. Auch im radiologischen Präsenzdienst werden manchmal eine unscharfe Diagnose und breite Differenzialdiagnosen der mutigen Festlegung vorgezogen. In der Entscheidungskette des Klinikers verliert das Problem der teleradiologischen Fehldiagnose allerdings nicht selten einen guten Teil der ihm sonst eigenen Brisanz.

\section{Wie wichtig ist das Urteil des Radiologen für den behandelnden Arzt?}

Aus seiner Gesamtverantwortung für die Versorgung des Patienten heraus ordnet der behandelnde Arzt die 
instrumentellen Untersuchungen nicht nur an, sondern er mustert die Resultate auch selbst und versucht, sich ein eigenes Urteil zu bilden. Die bildgebende Diagnostik wird dabei nicht anders behandelt als z.B. die Labormedizin, deren Ergebnisse ohne fachärztliche Befundinterpretation oder Deutungshilfe verwertet werden. Viele Fachärzte und Ärzte in fortgeschrittener Weiterbildung verfügen über gute bis sehr gute Kenntnisse in der bildgebenden Diagnostik ihrer Disziplin. Nicht nur die Vertreter z.B. der Pneumologie, der Orthopädie und Zahnmedizin, die zum Teil die Aufnahmen sogar selbst herstellen, legen täglich davon Zeugnis ab, sondern man kann diese Beobachtung auch und gerade in vielen Bereichen der Notfallmedizin, in der Traumatologie, Anästhesiologie und Neurologie/Neurochirurgie machen. Für den behandelnden Arzt ist das Urteil des Radiologen eine wesentliche, aber durchaus nicht die einzige und in der Praxis nicht immer die verbindliche und entscheidende Information über das Ergebnis der bildgebenden Diagnostik. Dafür ist die Radiologie zu wichtig.

\section{Wort- und Gedankenaustausch kann die klinische Diagnose und Therapie entscheidend beeinflussen}

Zudem hat die Entwicklung der Medizintechnik die Beurteilung des radiologischen Bildmaterials in den letzten Jahren erheblich beschleunigt und vereinfacht. Die Qualität der Aufnahmen ist, auch wenn sie von mehr oder weniger kooperationsfähigen Notfallpatienten stammen, in aller Regel befriedigend, vielfach sogar gut bis sehr gut. Artefakte nahezu jeglicher Provenienz werden inzwischen von vielen Geräten zum Teil selbständig eliminiert. Halb- oder vollautomatische 2D-, 3D- und 4D-Rekonstruktionen sind die Regel. Aus den grauen Schattenbildern der konventionellen Bildgebung sind durch die Rekonstruktionstechniken vielfarbige transparente Kunstwerke geworden. Jeder Arzt kann nach kurzer Einarbeitungszeit die Datensätze am Rechner selbständig bearbeiten. Softwareprogramme und andere Instrumente der computerassistierten Diagnostik liefern standardisierte multiparametrische Auswertungen mit Vorschlägen zur Differenzialdiagnose und ggf. Stadieneinteilung.

Mehrere radiologische Fachgesellschaften haben in den letzten Jahren ihre Mitglieder eindringlich dazu aufgerufen, die Befundungskonsolen häufiger als bisher zu verlassen und sich den klinischen Kollegen und den Patienten zuzuwenden, um auf diese Weise deutlicher als Entscheidungspartner wahrgenommen zu werden und agieren zu können. Die Teleradiologie ist für diese Offensive, ungeachtet ihrer anerkannten Vorzüge, auf den ersten Blick kein besonders gut geeignetes Wirkungsfeld. Dennoch kann sie auch und gerade dort, wo für sie strenge Vorschriften gelten, diese Initiativen fördern und für andere Regionen eine Vorbildfunktion entwickeln. Gemäss Röntgenverordnung § 3 Abs. 4 darf in Deutschland der Teleradiologe nur für Krankenhäuser innerhalb eines bestimmten Radius tätig werden, er muss die rechtfertigende Indikation prüfen, er muss das methodische Prozedere festlegen, und er muss in

Aus den grauen Schattenbildern der konventionellen Bildgebung sind durch die Rekonstruktionstechniken vielfarbige transparente Kunstwerke geworden.

der Lage sein, innerhalb des für die Notfallversorgung ausreichenden Zeitraums (45 bis 60 Minuten) vor Ort persönlich zu erscheinen. Zumeist kommuniziert der Teleradiologe - unabhängig von der Organisationsform des Diensts - seine Beurteilung nicht nur schriftlich, sondern teilt sie dem beauftragenden Arzt persönlich mit. Der Wort- und Gedankenaustausch kann die klinische Diagnose und die Therapie in vielen Fällen justieren oder sogar entscheidend beeinflussen und hilft, die möglichen Folgen von Fehldiagnosen zu vermeiden. Die mit der Kontaktaufnahme demonstrierte Empathie macht sich sowohl kurzfristig als auch auf Dauer bezahlt. Zugleich kann der Diagnostiker bei dieser Gelegenheit auch interventionelle Leistungen seiner Disziplin zur Diskussion stellen und, falls sich der behandelnde Arzt für eine davon entscheidet, zeitnah mit der Durchführung beginnen. So macht er sowohl gegenüber den Klinikern wie den Patienten eindrucksvoll deutlich, dass Teleradiologie nicht nur ein Notbehelf ist, sondern eine zukunftsträchtige Lösung sein kann - hierzulande ebenso wie anderswo.

\section{Literatur}

1 Howlett DC, Drinkwater K, Frost C, Higginson A, Ball C, Maskell G. The accuracy of interpretation of emergency abdominal CT in adult patients who present with non-traumatic abdominal pain: results of a UK national audit. Clin Radiol. 2017;72;41-51.

Bildnachweis

c Donfiore | Dreamstime.com

\section{Disclosure statement}

Der Autor deklariert keine Interessenbindungen in Zusammenhang mit diesem Artikel. 